\title{
Optimization Models and Algorithms for Services and Operations Management
}

\author{
Lu Zhen, ${ }^{1}$ Si Zhang, ${ }^{2}$ and Xinchang Wang ${ }^{3}$ \\ ${ }^{1}$ Shanghai University, Shanghai, China \\ ${ }^{2}$ George Mason University, Fairfax, VA, USA \\ ${ }^{3}$ Mississippi State University, Starkville, MS, USA \\ Correspondence should be addressed to Lu Zhen; lzhen@shu.edu.cn \\ Received 2 July 2017; Accepted 2 July 2017; Published 8 August 2017 \\ Copyright (C) 2017 Lu Zhen et al. This is an open access article distributed under the Creative Commons Attribution License, which \\ permits unrestricted use, distribution, and reproduction in any medium, provided the original work is properly cited.
}

Optimization of performances has achieved great success in industry and service domain. This way presents techniques for modeling real world decision problems and exhibits its ability to improve efficiency. Various types of algorithms are treated, with explanations on how they work and what their limitations are. We are devoted to creating, extending, and solving various models that arise in operations management of various industries. Not only are some developed models useful for theoretical research but also they can be immediately applied by practitioners. As all discussed models are problems of real world decision, various solution methods of the relevant problems are presented, including data mining technologies and intelligence algorithms. The most recent achievements are obtained in the field of optimization models and algorithms for services and operations management.

The articles contained in the present issue include the problems of industry and service domain. Most of them use mixed-integer linear programming models to solve the real world decision problems.

Teaching assistants (TAs) are essential in many departments in universities because of limited numbers of lecturers. So how the most appropriate teaching assistants will be assigned to the tutorials in a department? The contribution by X. Qu et al. "Mixed-Integer Linear Programming Models for Teaching Assistant Assignment and Extensions" provides a model about the objective to maximize the number of tutorials that are taught by the most suitable teaching assistants.

The tunnel safety issue has attracted more and more attention from the public as the development of the urban road tunnels. So how can the life cycle costs of tunnel safety provisions be minimized? In a paper by $\mathrm{P}$. Li and $\mathrm{X}$. Qu entitled "Optimal Allocation of Tunnel Safety Provisions Based on a Quantitative Risk Assessment Model," by taking advantage of the special structure of the optimization model, a Bi-Section Search and Bound Algorithm is designed to efficiently solve the problem.

Biofuel has become one of the most promising energies to replace fossil fuels in transportation sector, because of its various biomass and wide range of sources as well as huge potential for energy saving and emission reduction. But most of them have gone out of production due to both feedstock supply and biodiesel demand limitations until now. The paper by Y. Zhang et al. entitled "Robust Optimization on Regional WCO-for-Biodiesel Supply Chain under Supply and Demand Uncertainties" provides a way about how to study optimizing WCO-for-biodiesel supply chain with uncertainties to improve biorefineries' ability to cope with the poor environment.

In the EOQ inventory model with presale policy for deteriorating items, the demand rate depends on both onhand inventory and selling price. In the work by L. Zhao and J. You entitled "Optimal Pricing and Ordering Policy for Deteriorating Items with Stock-and-Price Dependent Demand and Presale Rebate," under the assumption that all the presale orders are fully backlogged with waiting-time dependent rebate, they derived optimal pricing and ordering policy by designing an effective algorithm.

Parallel machine systems are widely adopted in a variety of manufacturing environments, such as the semiconductor manufacturing industry and the electronics industry. 
The work by $\mathrm{H}$. Hu et al. entitled "Robust Parallel Machine Scheduling Problem with Uncertainties and SequenceDependent Setup Time" provides a method about the uniform parallel machine problem in the plastic production system, which involves uncertain processing time, job release time, and setup time for mold.

Product returns have been one of the main sources resulting in inefficiency in Internet retail market and managers are continuously striving for a more scientific and efficient network layout to arrange the returned goods. In the paper by X. Wang et al. entitled "A Network Optimization Research for Product Returns Using Modified Plant Growth Simulation Algorithm," the authors proposed a mixed-integer nonlinear programming model with the aim of minimizing total cost and created a high-efficiency method, the Modified Plant Growth Simulation Algorithm (MPGSA), to optimize the problem.

Reasonable purchasing price and good quality of raw materials can reduce manufacturing cost in supply chain network. In the paper by $\mathrm{H}$. Hu et al. entitled "A Mixed Integer Programming Model for Supplier Selection and Order Allocation Problem with Fuzzy Multiobjective," the authors showed that three objectives have different weights which are given by experts. The method of fuzzy analytic hierarchy process (FAHP) is used to calculate the weighted values. And the result shows that a weighted model is more advantageous for supplier selection and order allocation.

The effective arrangements for the supporting operations of aircrafts in flight deck are critical to the aircraft carrier. The paper by L. Yu et al. entitled "An Extended Flexible Job Shop Scheduling Model for Flight Deck Scheduling with Priority, Parallel Operations, and Sequence Flexibility" describes a novel mixed integer liner programming formulation (MILP) for the flight deck scheduling problem. The results show that their algorithm can meet both the operational relations and the takeoff priority requirements.

The significant problem is about the key resource scheduling of container terminals for energy-efficient operation in terminal management. In the paper by D. Chang et al. entitled "Defining Scheduling Problems for Key Resources in EnergyEfficient Port Service Systems," an energy-efficient evaluation model of the key resource scheduling is proposed, and constraint set of key resource scheduling of a container terminal for energy-efficient operation is established in this paper.

With the international container, transportation has experienced rapid growth. Yard crane equipment and yard space are the key resources in the container terminal yard. In the paper by C. Tan and J. He entitled "Integrated Yard Space Allocation and Yard Crane Deployment Problem in Resource-Limited Container Terminals," the authors studied the integrated yard space allocation and yard crane deployment problem in resource-limited container terminals where yard space and yard cranes are extremely scarce.

In order to get quicker than the standard situation in detecting small and moderate shifts in the process of service and management operation, the paper by S. Yu et al. entitled "Statistical Design of an Adaptive Synthetic $\bar{X}$ Control Chart with Run Rule on Service and Management Operation" provides an improved synthetic control chart based on hybrid adaptive scheme and run rule scheme is introduced to enhance the statistical performance of traditional synthetic control chart on service and management operation.

Operations management has become one of the most widely explored researching areas. In the paper by H. Fei et al. entitled "A Survey of Recent Research on Optimization Models and Algorithms for Operations Management from the Process View," the purpose is to give an overview of optimization modeling and resolution approaches, which are applied to operations management.

We hope that the application of the optimization model in industry and service domain will serve the services and operations management.

\section{Acknowledgments}

We would like to express our appreciation to all the authors for their informative contributions and the reviewers for their support and constructive critiques in making this special issue possible.

Lu Zhen Si Zhang Xinchang Wang 

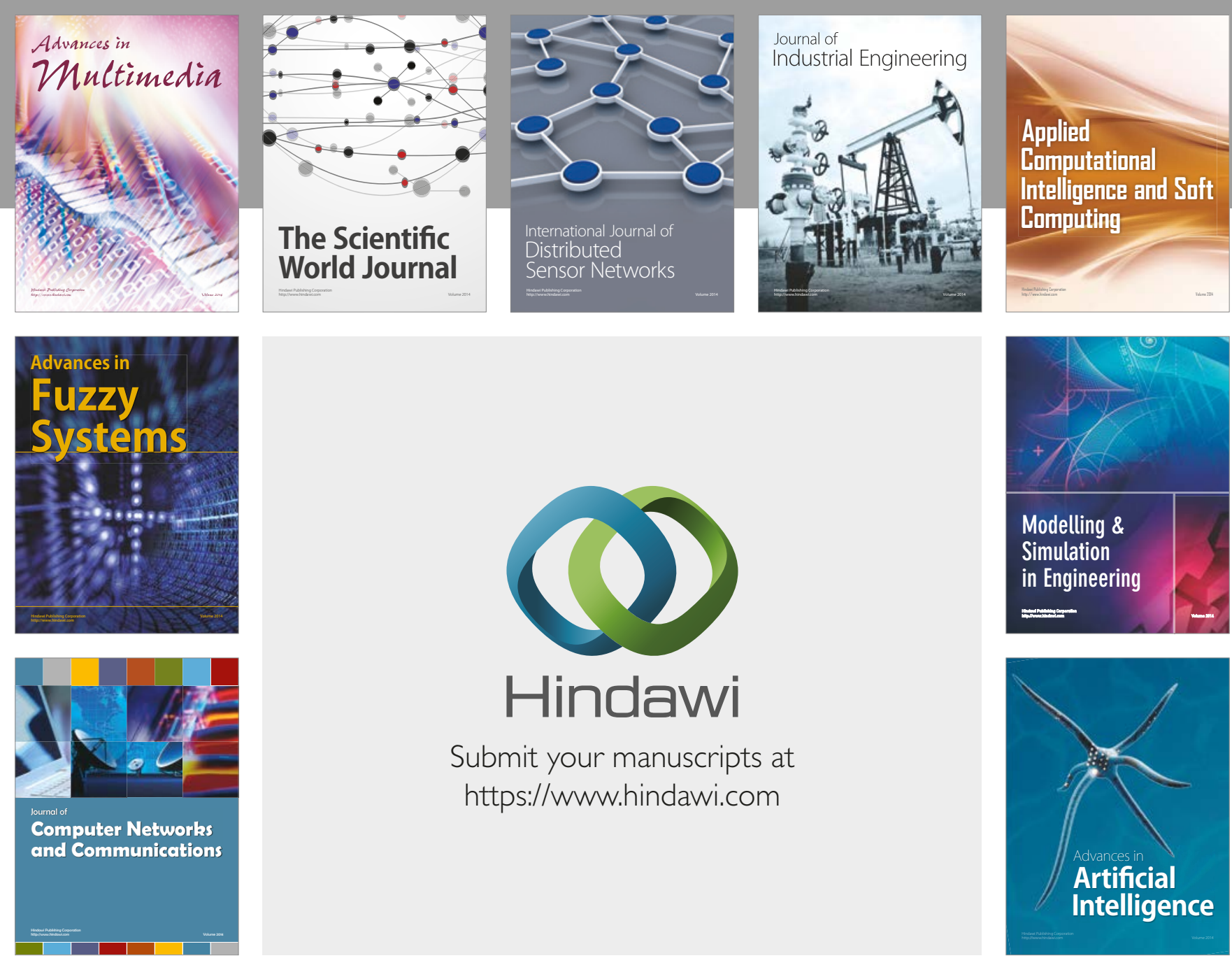

\section{Hindawi}

Submit your manuscripts at

https://www.hindawi.com
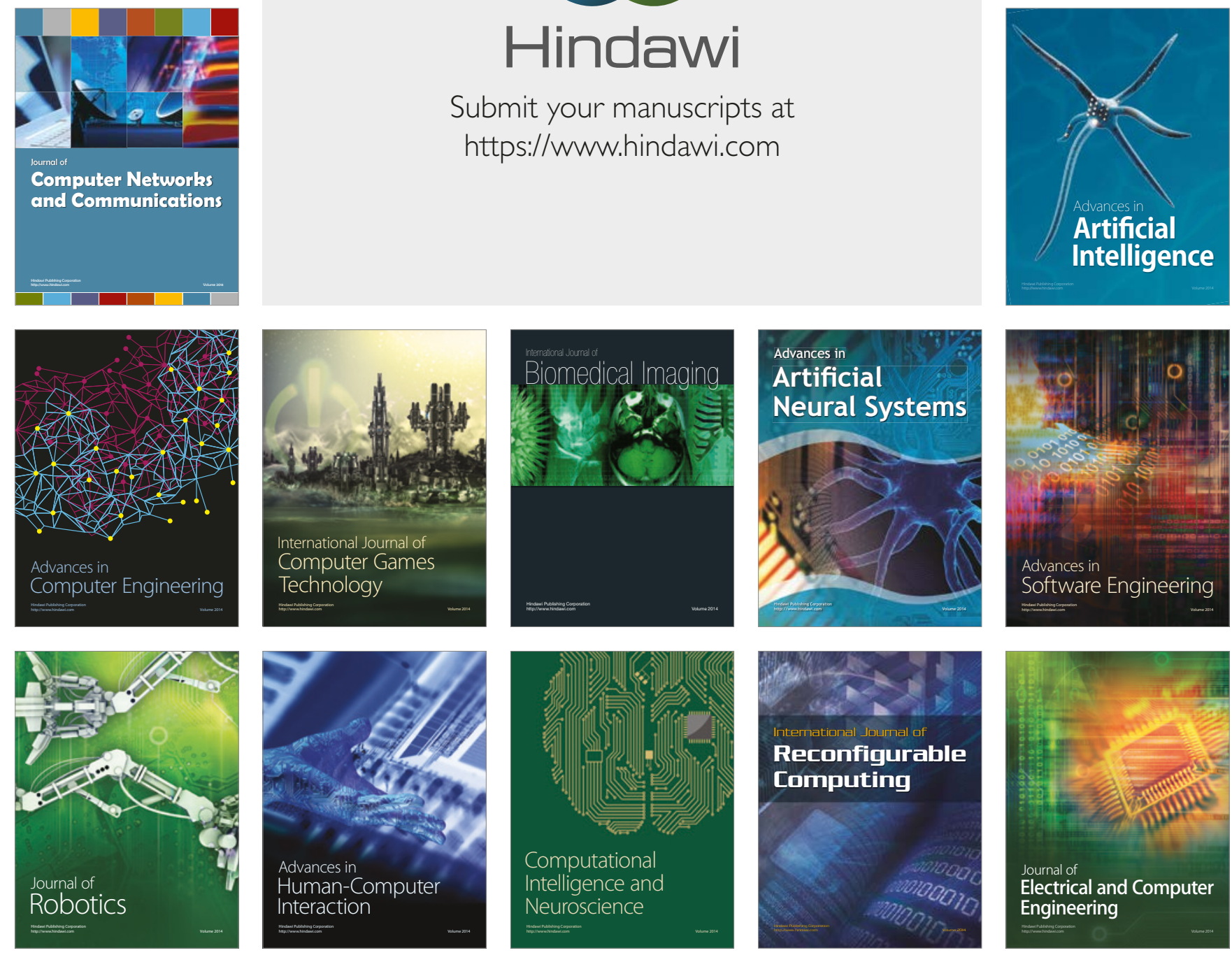